\title{
Repolarization of Polarization-Scrambled Optical Signals Due to Polarization Dependent Loss
}

\author{
C. R. Menyuk, Senior Member, IEEE, D. Wang, and A. N. Pilipetskii
}

\begin{abstract}
We calculate the distribution function of the degree of polarization of an initially polarization scrambled signal propagating long distances in an optical fiber. We show that a significant repolarization can occur for modern-day parameters. We estimate how far the polarization dependent loss must be reduced before repolarization becomes negligible.
\end{abstract}

Index Terms - Birefringence, Fokker-Planck equations, optical fiber amplifiers, optical fiber communication, polarization, stochastic differential equations.

\section{INTRODUCTION}

$\mathbf{T}$ HE COMBINATION of polarization dependent loss, polarization dependent gain, and polarization mode dispersion can lead to fading, which degrades the performance of high-data-rate long-distance communication systems [1]-[5]. Polarization dependent gain impacts the performance by causing excess noise in the polarization state orthogonal to the signals. Polarization mode dispersion in combination with polarization dependent gain leads to amplitude crosstalk between channels in a wavelength-division multiplexed system. Both polarization dependent gain and polarization mode dispersion tend to depolarize a signal and will in principle pose little or no difficulty if the input signal is depolarized. Therefore, recent experiments have used polarization scrambling in which all the Stokes parameters average to zero on a time scale that is short compared to the response time of the erbium-doped fiber amplifiers [6], [7], e.g., a single bit. By contrast, polarization dependent loss tends to repolarize a signal. Thus, not only does polarization dependent loss directly degrade a communication signal, but, by repolarizing an initially polarization-scrambled signal, it can seed further degradations due to polarization dependent gain and polarization mode dispersion.

In this letter, we will consider the simple case of a single channel in which polarization dependent loss elements located at amplifiers alternate with lengths of optical fiber, as shown schematically in Fig. 1. We will derive an analytical formula for the probability distribution function of the degree-ofpolarization and compare the formula to Monte Carlo simulations. We will show that if each amplifier has a polarization dependent loss of $x_{\mathrm{PDL}}=0.1 \mathrm{~dB}$, which is a typical value

Manuscript received March 19, 1997; revised May 9, 1997. This work was supported by AT\&T, by the Defense Advanced Research Projects Agency through the Air Force Office of Scientific Research, the U.S. Department of Energy, and the National Science Foundation.

The authors are with the Department of Computer Science and Electrical Engineering, University of Maryland Baltimore County, Baltimore, MD 21250 USA.

Publisher Item Identifier S 1041-1135(97)06368-4.

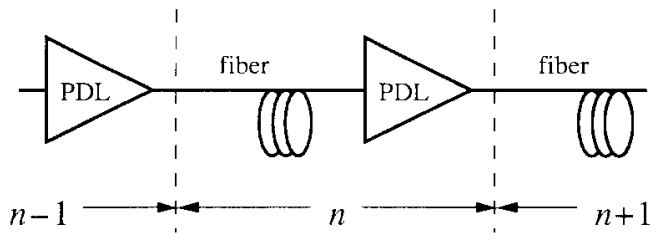

Fig. 1. Schematic illustration of the model configuration.

for current systems [5], [8], then a significant amount of repolarization will occur. We will then discuss the scaling of the repolarization with $x_{\text {PDL }}$ and the implications for communication systems. The model that we will use follows the Stokes parameters of the channel and does not take into acount dispersion and nonlinearity. Since polarization dependent loss affects the whole signal uniformly, we do not believe that the impact of this simplification is large. Also, we do not include the polarization dependent gain and the polarization mode dispersion since the goal of this letter is to calculate the repolarization due to polarization dependent loss and show that it can be significant, rather than attempting to calculate the overall penalty due to all polarization effects at once.

\section{THEORY}

The effect of a polarization dependent loss element is to cause excess loss in one of two orthogonal polarizations. Using the Jones vector notation where we take the seconf component to be in the direction of maximum loss, we may write

$$
\left(\begin{array}{l}
u_{1} \\
u_{2}
\end{array}\right)_{\text {after }}=\left(\begin{array}{ll}
1 & 0 \\
0 & \alpha
\end{array}\right)\left(\begin{array}{l}
u_{1} \\
u_{2}
\end{array}\right)_{\text {before }} .
$$

The quantities $u_{1}$ and $u_{2}$ are the complex field amplitudes, and $\alpha$ is related to $x_{\text {PDL }}$ through the relationship, $x_{\text {PDL }}$ (in $\mathrm{dB})=-20 \log _{10} \alpha$.

Since we are interested in the repolarization of an entire communication channel, we will be focusing on the evolution of the Stokes parameters

$$
\begin{aligned}
S_{0} & =\frac{1}{2 T} \int_{-T}^{T}\left[\left|u_{1}(t)\right|^{2}+\left|u_{2}(t)\right|^{2}\right] d t \\
S_{1} & =\frac{1}{2 T} \int_{-T}^{T}\left[\left|u_{1}(t)\right|^{2}-\left|u_{2}(t)\right|^{2}\right] d t \\
S_{2} & =\frac{1}{T} \int_{-T}^{T} \Re\left[u_{1}(t) u_{2}^{*}(t)\right] d t \\
S_{3} & =\frac{1}{T} \int_{-T}^{T} \Im\left[u_{1}(t) u_{2}^{*}(t)\right] d t
\end{aligned}
$$


where we are assuming that $T$ is very large compared to a single bit period and that the channel becomes statistically stationary when $T$ is large so that this definition is meaningful. From (1) and (2), we find

$$
\begin{aligned}
S_{0, \text { after }} & =\frac{1+\alpha^{2}}{2} S_{0, \text { before }}+\frac{1-\alpha^{2}}{2} S_{1, \text { before }} \\
S_{1, \text { after }} & =\frac{1-\alpha^{2}}{2} S_{0, \text { before }}+\frac{1+\alpha^{2}}{2} S_{1, \text { before }} \\
{\left[S_{2}+j S_{3}\right]_{\text {after }} } & =\alpha\left[S_{2}+j S_{3}\right]_{\text {before }}
\end{aligned}
$$

The effect of the fiber between the polarization dependent loss elements is to randomly rotate the Stokes vector $S_{\mathrm{pol}}=$ $\left(S_{1}, S_{2}, S_{3}\right)$ on the Poincaré sphere. We thus obtain the following iterative equations,

$$
\begin{aligned}
S_{0}^{(n+1)} & =\frac{1+\alpha^{2}}{2} S_{0}^{(n)}+\frac{1-\alpha^{2}}{2} S_{\mathrm{pol}} \cos \theta^{(n)} \\
S_{1}^{(n+1)} & =\frac{1-\alpha^{2}}{2} S_{0}^{(n)}+\frac{1+\alpha^{2}}{2} S_{\mathrm{pol}} \cos \theta^{(n)} \\
{\left[S_{2}+j S_{3}\right]^{(n+1)} } & =\alpha S_{\mathrm{pol}}^{(n)} \sin \theta^{(n)} \exp \left(j \phi^{(n)}\right)
\end{aligned}
$$

where

$$
\left(S_{\text {pol }}^{(n)}\right)^{2}=\left(S_{1}^{(n)}\right)^{2}+\left(S_{2}^{(n)}\right)^{2}+\left(S_{3}^{(n)}\right)^{2} .
$$

The $\cos \theta^{(n)}$ are independent and identically distributed (i.i.d.) random variables, uniformly distributed in the range $[-1,1]$ so that $\left\langle\cos ^{2} \theta^{(n)}\right\rangle=1 / 3$. Similarly, the $\phi^{(n)}$ are i.i.d. random variables that are also independent of the $\cos \theta^{(n)}$ and are uniformly distributed in the range $[0,2 \pi]$. Equations (4) and (5) together constitute a random process, and our goal is to solve them simultaneously in order to determine $f\left(d_{\text {pol }}^{(n)}\right)$, the probability distribution function for $d_{\mathrm{pol}}^{(n)}=S_{\mathrm{pol}}^{(n)} / S_{0}^{(n)}$.

Equations (4) and (5) do not take into account polarization independent loss and gain. In reality, the amplifiers in optical fiber telecommunication systems are set to operate in saturation so that the total power $S_{0}^{(n)}$ is maintained as close to constant as possible [9]. Since $d_{\mathrm{pol}}^{(n)}$ is not affected by the addition of polarization independent loss and gain, we do not include it, but the reader should be aware that $S_{0}^{(n)}$ and $S_{\text {pol }}^{(n)}$ are not separately meaningful. Only the ratio $S_{\text {pol }}^{(n)} / S_{0}^{(n)}=d_{\text {pol }}^{(n)}$ is meaningful. Since $d_{\mathrm{pol}}^{(n)}$ is not affected by the total power level, we will simply set $S_{0}^{(0)}=1$. We also set $S_{\text {pol }}^{(0)}=0$, which is equivalent to assuming $d_{\mathrm{pol}}^{(0)}=0$, so that the channel is initially polarization scrambled.

To make further theoretical progress, we next eliminate $\cos \theta^{(n)}$ and $\phi^{(n)}$ from (4) and (5), obtaining

$$
\begin{aligned}
\left(S_{0}^{(n+1)}\right)^{2}-\left(S_{\text {pol }}^{(n+1)}\right)^{2} & =\alpha^{2}\left[\left(S_{0}^{(n)}\right)^{2}-\left(S_{\text {pol }}^{(n)}\right)^{2}\right] \\
& =\alpha^{2(n+1)}
\end{aligned}
$$

This relation suggests replacing $S_{0}^{(n)}$ and $S_{\text {pol }}^{(n)}$ with the new variables $x^{(n)}=S_{0}^{(n)} / \alpha^{n}$ and $y^{(n)}=S_{\text {pol }}^{(n)} / \alpha^{n}$, which now obey the equations

$$
\begin{gathered}
x^{(n+1)}=\frac{1+\alpha^{2}}{2 \alpha} x^{(n)}+\frac{1-\alpha^{2}}{2 \alpha} y^{(n)} \cos \theta^{(n)} \\
\left(x^{(n)}\right)^{2}-\left(y^{(n)}\right)^{2}=1
\end{gathered}
$$

where $d_{\mathrm{pol}}^{(n)}=y^{(n)} / x^{(n)}$. Combining (7a) and (7b) yields

$$
x^{(n+1)}=\frac{1+\alpha^{2}}{2 \alpha} x^{(n)}+\frac{1-\alpha^{2}}{2 \alpha}\left[\left(x^{(n)}\right)^{2}-1\right]^{1 / 2} \cos \theta^{(n)} .
$$

Since $\alpha$ is close to one, the change in each step is small, and it is reasonable to replace (8) by the stochastic differential equation

$$
\dot{x}=\beta x+\left(x^{2}-1\right)^{1 / 2} \zeta
$$

where $\dot{x}=d x / d n$ is the first-order stochastic differential operator, $\zeta$ is a white noise process with variance $\sigma_{\zeta}^{2}=$ $\left(1-\alpha^{2}\right)^{2} / 12 \alpha^{2}$, and $\beta=(1-\alpha)^{2} / 2 \alpha[10] .{ }^{1}$ Since (8) is a forward difference equation, (9) must be interpreted in the sense of Ito [10], ${ }^{2}$ which implies that (9) represents a diffusion process, and the evolution of the distribution function of $x$, $f_{x}(x)$, is governed by the Fokker-Planck equation

$$
\frac{\partial f_{x}}{\partial n}+\beta \frac{\partial}{\partial x} x f_{x}-\frac{\sigma_{\zeta}^{2}}{2} \frac{\partial^{2}}{\partial x^{2}}\left(x^{2}-1\right) f_{x}=0 .
$$

Changing variables from $x$ to $\gamma$, where $x=\cosh \gamma$, one finds that $f_{\gamma}(\gamma)=f_{x}[x(\gamma)] d x / d \gamma=f_{x}[x(\gamma)] / \sinh \gamma$ is governed by the Fokker-Planck equation

$$
\frac{\partial f_{\gamma}}{\partial n}+\left(\beta-\frac{1}{2} \sigma_{\zeta}^{2}\right) \frac{\partial}{\partial \gamma} \operatorname{coth}(\gamma) f_{\gamma}-\frac{1}{2} \sigma_{\zeta}^{2} \frac{\partial^{2} f_{\gamma}}{\partial \gamma^{2}}=0
$$

where $d_{\text {pol }}=\tanh \gamma$. In the limit of interest to us in which $\gamma \lesssim 1$, one may replace $\operatorname{coth} \gamma \simeq \gamma^{-1}$, in which case (11) has the solution

$$
\begin{aligned}
f_{\gamma}(\gamma)= & \frac{2}{\left(2 \sigma_{\zeta}^{2} n\right)^{1 / 2} \Gamma\left(\beta / \sigma_{\zeta}^{2}\right)}\left(\frac{\gamma^{2}}{2 \sigma_{\zeta}^{2} n}\right)^{\left(\beta / \sigma_{\zeta}^{2}\right)-(1 / 2)} \\
& \cdot \exp \left(-\frac{\gamma^{2}}{2 \sigma_{\zeta}^{2} n}\right) \\
\simeq & \frac{4}{\pi^{1 / 2}} \frac{\gamma^{2}}{\left(2 \sigma_{\zeta}^{2} n\right)^{3 / 2}} \exp \left(-\frac{\gamma^{2}}{2 \sigma_{\zeta}^{2} n}\right)
\end{aligned}
$$

with $\beta / \sigma_{\zeta}^{2}=6 \alpha /(1+\alpha)^{2} \simeq 3 / 2$. From (12), one may obtain $f\left(d_{\text {pol }}\right)$ using the relationship

$$
f\left(d_{\mathrm{pol}}\right)=f_{\gamma}(\gamma) d \gamma / d\left(d_{\mathrm{pol}}\right)
$$

Fig. 2 compares the analytical expression for $f\left(d_{\text {pol }}\right)$ from (12) and (13) to a Monte Carlo solution of (4) and (5), setting $x_{\mathrm{PDL}}=0.1$. We used $10^{6}$ representations of the $\cos \theta^{(n)}$. Two points are apparent. The first is that the theory is indistinguishable from the simulation. The second is that when $n=300$, corresponding to transoceanic distances, a significant amount of repolarization occurs.

${ }^{1}$ In [10], (4.1.1) and the surrounding discussion define Ito stochastic differential equations. Equation (10.3.1) and the subsequent discussion show that forward difference equations like (8) should be approximated by stochastic differential equations interpreted in the sense of Ito (as opposed to Stratonovich)

${ }^{2}$ Diffusion processes are defined and the Fokker-Planck equation is derived in [10, Secs. 2.5, 2.6, ch. 9]. 


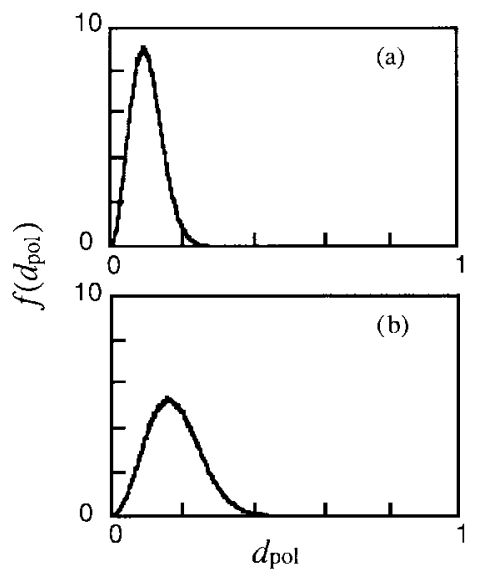

Fig. 2. Comparison of the distribution function $f\left(d_{\text {pol }}\right)$ obtained by Monte Carlo simulation of the original difference equations [(4) and (5)] to the theoretically calculated function [(12) and (13)]. The parameters are $x_{\mathrm{PDL}}=$ 0.1 . (a) $n=100$. (b) $n=300$. The two approaches yield indistinguishable results.

\section{DISCUSSION}

A thorough discussion of the impact that the repolarization has on long-distance, high-data rate systems must await the development of a more complete model that includes polarization dependent gain and polarization mode dispersion. Nonetheless, it is possible to immediately draw from this work a number of simple results that are useful for system design. First, polarization dependent loss can lead to a significant repolarization of a polarization scrambled signal. This repolarization can then seed further signal degradation due to polarization dependent gain and polarization mode dispersion; so, there is a clear motivation to reduce $x_{\mathrm{PDL}}$ as much as possible. From (12), using $d_{\mathrm{pol}} \simeq \gamma$, we find $d_{\mathrm{pol}, \max } \simeq 0.09 n^{1 / 2} x_{\mathrm{PDL}}$ and $\left\langle d_{\text {pol }}\right\rangle=0.11 n^{1 / 2} x_{\text {PDL }}$, where $d_{\text {pol,max }}$ is the point at which $f\left(d_{\mathrm{pol}}\right)$ attains its maximum, and $\left\langle d_{\mathrm{pol}}\right\rangle$ is the mean of $f\left(d_{\mathrm{pol}}\right)$. For the example presented in Fig. 2(b), in which $n=300$ and $x_{\text {PDL }}=0.1$, we find that $d_{\text {pol, } \max }=0.16$ and $\left\langle d_{\text {pol }}\right\rangle=0.19$.

How low must we make $x_{\text {PDL }}$ before it can be neglected entirely? We may estimate this value very roughly as follows: After polarization scrambling, the residual $d_{\text {pol }}$ is about $15 \%$ [8]; so, it is reasonable to assume that if the additional repolarization is under $15 \%$, then it can be neglected. If we assume that a fiber cable has a lifetime of 20 years and, somewhat conservatively, that it changes its polarization state every hour so that a different value of $d_{\text {pol }}$ is sampled, then the cable passes through $2 \times 10^{5}$ states in its lifetime. Thus, if the probability of obtaining a repolarization of 0.15 is less than $5 \times 10^{-6}$, then repolarization can be ignored. Integrating (12), we find that $d_{\text {pol }} \gtrsim 35 n^{1 / 2} x_{\text {PDL }}$ with probability $5 \times$ $10^{-6}$; so, with $n=300$, our criterion becomes $x_{\mathrm{PDL}} \lesssim 0.025$ which is about a quarter of the current best value. While this sort of reduction of $x_{\mathrm{PDL}}$ may be difficult to obtain, it seems worth striving for.

\section{ACKNOWLEDGMENT}

One of the authors, C. R. Memyuk, would like to acknowledge useful technical discussions with a number of past and current members of Submarine Lightsystems Inc. at AT\&T-in particular, N. Bergano, F. Kerfoot, and E. Lichtman. He thanks M. Shtaif for a careful reading of this manuscript. The authors thank P. Runge for encouraging this project.

\section{REFERENCES}

[1] C. D. Poole, R. W. Tkach, A. R. Chraplyvy, and D. A. Fishman, "Fading in lightwave systems due to polarization-mode dispersion," IEEE Photon. Technol. Lett., vol. 3, pp. 68-70, Jan. 1991.

[2] S. Yamamoto, N. Edagawa, H. Taga, Y. Yoshida, and H. Wakabayashi, "Observation of BER degradation due to fading in long distance optical amplifer system," Electron. Lett., vol. 29, no. 2, pp. 209-210, 1993.

[3] F. Bruyére and O. Audouin, "Assessment of system penalties induced by polarization mode dispersion in a $5 \mathrm{~Gb} / \mathrm{s}$ optically amplified transoceanic link," IEEE Photon. Technol. Lett., vol. 6, pp. 443-445, Mar. 1994.

[4] E. Lichtman, "Performance limitations imposed on all-optical ultralong lightwave systems at the zero-dispersion wavelength," J. Lightwave Technol., vol. 13, pp. 898-905, May 1995.

[5] _ "Limitations imposed by polarization-dependent gain and loss on all-optical ultralong communication systems," J. Lightwave Technol., vol. 13, pp. 906-913, May 1995.

[6] N. S. Bergano, C. R. Davidson, and F. Heismann, "Bit-synchronous polarization and phase modulation scheme for improving the performance of optical amplifier transmission systems," Electron. Lett., vol. 32, no. 1, pp. 52-54, 1996.

[7] N. S. Bergano and C. R. Davidson: "Wavelength division multiplexing in long-haul transmission systems," J. Lightwave Technol., vol. 14, pp. 1299-1308, June 1996.

[8] N. S. Bergano, private communication.

[9] J. Schesser, S. M. Abbott, R. L. Easton, and M. S. Stix, "Design requirements for the current generation of undersea cable systems," AT\&T Tech. J., vol. 74, no. 1, pp. 16-32, 1995.

[10] L. Arnold, Stochastic Differential Equations: Theory and Applications. New York: Wiley, 1974. 